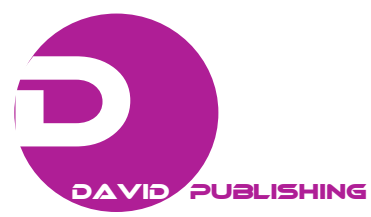

\title{
Periodic Precipitation of Liesegang System under Microwave Radiation
}

\author{
Yushin Kanazawa and Yusuke Asakuma* \\ Department of Mechanical and System Engineering, University of Hyogo, Himeji 671-2280, Japan
}

Received: February 27, 2014 / Accepted: March 15, 2014 / Published: April 25, 2014.

\begin{abstract}
We studied precipitation patterns in a Liesegang system under MW (microwave) irradiation in order to investigate metal salt diffusion in an electrolyte gel. The gel and salt concentrations were varied. MW irradiation induced periodic patterns of precipitation because polar molecules vibrate and rotate in an electromagnetic field. For example, the number of patterns increased by the irradiation. Accordingly, microwave irradiation nonlinearly accelerated the diffusion of ionic molecules.
\end{abstract}

Key words: MW, Liesegang, diffusion, precipitation.

\section{Introduction}

Liesegang phenomena, a function of diffusion and reaction conditions, form attractive and unique periodic stripe patterns. Such pattern formation frequently inspires microstructural design in materials science. Moreover, morphological pattern transitions between periodic precipitation and tree-like crystal aggregates are scientifically interesting $[1,2]$. Liesegang phenomena usually manifest themselves when a concentrated salt solution diffuses through a gel that contains another electrolyte at a lower concentration. Diffusion and chemical reactions cause little precipitation of otherwise soluble salts in a periodic manner. The precipitation and redissolution reactions for a generic metal ion $\mathrm{M}^{2+}$ are as follows:

$$
\begin{gathered}
\mathrm{M}^{2+}(\mathrm{aq})+2 \mathrm{NH}_{4} \mathrm{OH}(\mathrm{aq}) \rightarrow 2 \mathrm{NH}_{4}^{+}(\mathrm{aq})+\mathrm{M}(\mathrm{OH})_{2}(\mathrm{~s}) \\
\mathrm{M}(\mathrm{OH})_{2}(\mathrm{~s})+6 \mathrm{NH}_{4}^{+}(\mathrm{aq}) \rightarrow \\
\mathrm{M}\left(\mathrm{NH}_{3}\right)_{6}{ }^{2+}(\mathrm{aq})+2 \mathrm{H}_{2} \mathrm{O}(\mathrm{l})+4 \mathrm{H}^{+}(\mathrm{aq})
\end{gathered}
$$

When $\mathrm{NH}_{4} \mathrm{OH}$ diffusion causes metal ions to precipitate in the form of $\mathrm{M}(\mathrm{OH})_{2}$, redissolution at the top of the pattern induces a Liesegang band.

Recent research indicates that spontaneous

${ }^{*}$ Corresponding author: Yusuke Asakuma, Associate Professor, research fields: heat and mass transfer, microwave. E-mail: asakuma@eng.u-hyogo.ac.jp. diffusion-related self-organization dynamics and irreversible reactions or precipitation systems based on Ostwald supersaturation theory explain Liesegang pattern formation [3]. However, nonlinearity hinders elaboration of the exact mechanism.

MW (microwave) radiation may be helpful in this context. MW radiation has recently been a very active area of research due to the many advantages of nonthermal effects such as reaction promotion, nucleation induction, and diffusion facilitated by the molecular vibration of polar molecules $[4,5]$. We hypothesized that the reaction and diffusion characteristics of a Liesegang system could be modulated or explained via various MW properties. In addition, Liesegang phenomena may suggest a new application of MW radiation and suitable operating conditions for highly functional inorganic nanomaterials. Salt and gel concentration are essential for solving the diffusion challenge of Liesegang phenomena. In this study, we varied gel and salt concentrations to understand the MW effect. We used a MW reactor equipped with an in situ observation system to analyze the dynamic growth behavior of the periodic pattern. 


\section{Experiments}

To form a Liesegang pattern, we used a gel because it can prevent precipitate sedimentation and hydrodynamic turbulence. A salt was added to a prepared gelatin-based gel $[6,7]$. In this study, gelatin and salt concentrations, $C_{g}, C_{c}$ were varied, and we used $\mathrm{Co}\left(\mathrm{NO}_{3}\right)_{2}$ (Table 1). We first heated a solution (i.e., gel and salt) to $90{ }^{\circ} \mathrm{C}$. We then poured the solution into a glass test tube $(\Phi: 13 \mathrm{~mm}$; height: $100 \mathrm{~mm}$; volume: $5 \mathrm{~mL}$ ) and gradually cooled it to room temperature to impart a homogeneous gelatin network structure. Temperature is the most important factor for pattern formation; we used two different methods, MW irradiation and conventional heating (OB, oil bath) for maintaining solution temperature. After we poured $2 \mathrm{~mL}$ of a $28 \%$ ammonium solution at the gel surface, we covered it with a silicon rubber lid. Ammonium salt precipitates via ammonium ion diffusion into the gel, and Liesegang stripe-pattern formation starts from the top surface to the bottom of the tube. We measured the stripe number and length for the two heating methods.

\subsection{MW Heating}

Fig. 1 shows a tube-guide MW reactor (Shikoku Instrumentation), which is specially designed for preventing MW leakage and equipped with a microscope camera (Sigma Koki, model SK-TC202USB-AT) for in situ observation. We hung a test tube containing the solution by a thread at the

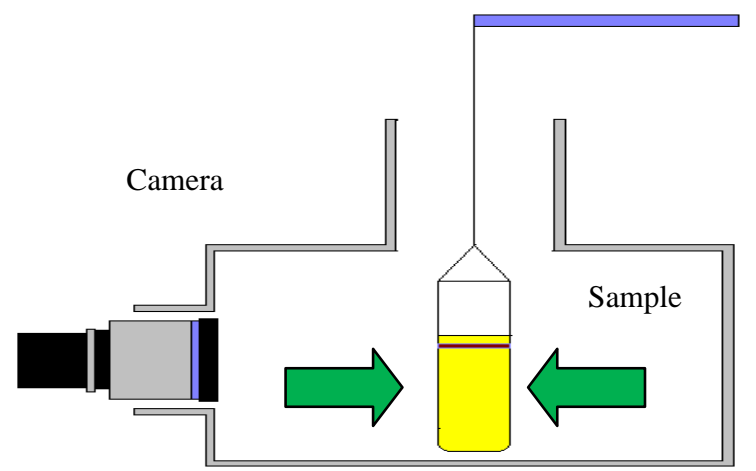

(a) Side view center of the reactor. Immediately after pouring the ammonium solution onto the gel surface, we initiated MW irradiation for $30 \mathrm{~min}$. Because the stripe pattern grows from the surface to the bottom, we gradually lifted up the test tube at a controlled speed to acquire photographs around the vertical stripe center, through the side of the reactor, with the fixed microscope. We measured the Liesegang pattern length via the pixel number between the top and bottom of the precipitation region, and we counted the stripes through the photographs every 5 min. This approach enables dynamic evaluation of Liesegang pattern propagation with respect to MW radiation. After 30 min, we measured the final temperature with an optical fiber (Anritsu Meter) because the solution temperature increases under irradiation.

\subsection{Conventional Heating}

We used an $\mathrm{OB}$ to maintain the solution temperature. The bath temperature was increased over the course of $30 \mathrm{~min}$ to the final temperature observed in the MW experiment. We poured ammonium solution into the test tube immersed in the $\mathrm{OB}$, and photographed the stripe pattern after 30 min with the microscope camera.

\section{Table 1 Experimental conditions.}

\begin{tabular}{llll}
\hline & $\begin{array}{l}\text { Gel concentrate } \\
C_{g}(\mathrm{wt} \%)\end{array}$ & $\begin{array}{l}\text { Salt concentrate } \\
C_{c}(\mathrm{~mol} / \mathrm{L})\end{array}$ & $\begin{array}{l}\text { MW output (W) } \\
\text { or temperature } \\
\left({ }^{\circ} \mathrm{C}\right)\end{array}$ \\
\hline $\mathrm{MW}$ & $0.5,1,2$ & $0.3,0.6,0.9$ & $25(\mathrm{~W})$ \\
$\mathrm{OB}$ & $0.5,1,2$ & $0.3,0.6,0.9$ & $40\left({ }^{\circ} \mathrm{C}\right)$ \\
\hline
\end{tabular}

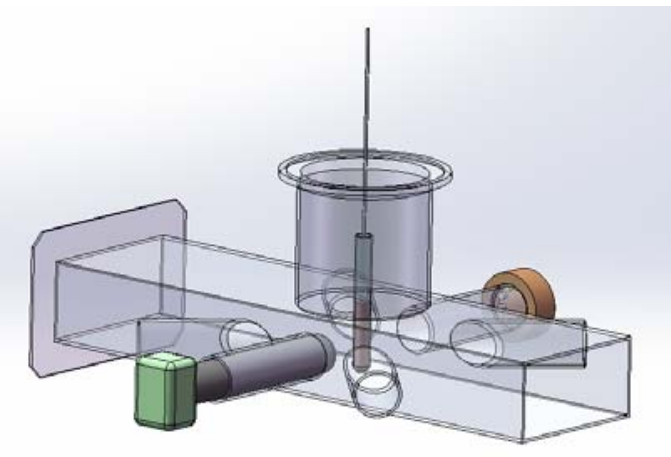

(b) 3D view

Fig. 1 MW reactor. 


\section{Results and Discussion}

Figs. 2 and 3 show photographs of the stripe pattern near the gel surface for each gel concentration under MW or OB conditions after $30 \mathrm{~min}$. The experiment involves precipitation after the salt concentration in the gel exceeds the critical values (i.e., the solubility due to ammonium ion diffusion). Although a stripe pattern was observed, an expected pattern transition (e.g., a tree-like pattern [1]) was not observed in this experiment. On the other hand, the parallel stripe pattern remained even under MW irradiation.

To aid in understanding the mechanism of stripe-pattern formation, Figs. 4 and 5 plot the stripe number and length, respectively, in relation to reaction time. Filled and open symbols indicate MW and $\mathrm{OB}$ conditions, respectively. Although precipitation starts just after contact with the ammonium solution, either the stripes do not immediately form or the camera resolution limit precludes sufficiently fast and precise image acquisition; accordingly, we assigned the initial number of stripes as 0 . We counted the number of stripes after approximately 15-25 min. The stripe length linearly increased with reaction time (Fig. 5). After $30 \mathrm{~min}$ of reaction time, nonthermal effects induced by MW irradiation caused the stripe number and length to increase more rapidly for MW relative to OB. This is attributable to enhanced molecular diffusion and facilitated supersaturation. Molecular vibration induced by MW irradiation accelerates ionic diffusion. Moreover, such vibration readily induces supersaturation of the precipitate. Consequently, cyclic periods of supersaturation, nucleation, and depletion become faster. However, temperature must be carefully controlled to modulate such cycling.

Figs. 6 and 7 show an almost linear relation of stripe number and length, after 30 min of irradiation, with respect to $\mathrm{Co}\left(\mathrm{NO}_{3}\right)_{2}$ concentration. $\mathrm{MW}$ imparted a greater effect than $\mathrm{OB}$ in case of the lower salt concentration. Consequently, MW irradiation more effectively induced precipitation via supersaturation.
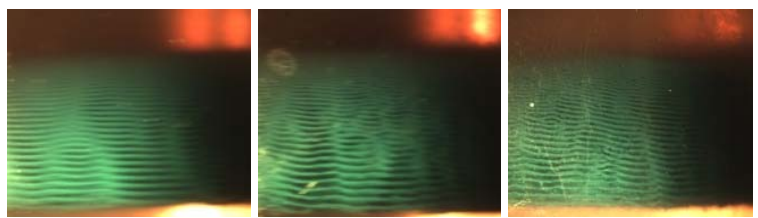

(a) $C_{g}=0.5 \mathrm{wt} \%$

(b) $C_{g}=1.0 \mathrm{wt} \%$

(c) $C_{g}=2.0 \mathrm{wt} \%$

Fig. 2 Stripe pattern under $\mathrm{MW}$ at $C_{c}=0.3 \mathrm{~mol} / \mathrm{L}$.

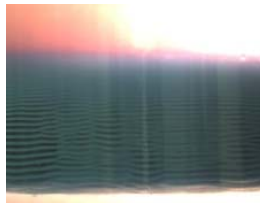

(a) $C_{g}=0.5 \mathrm{wt} \%$

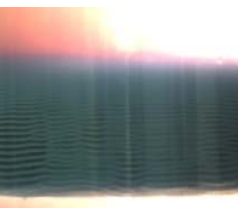

(b) $C_{g}=1.0 \mathrm{wt} \%$

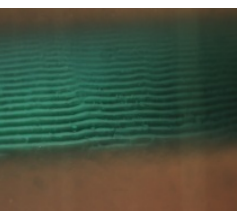

(c) $C_{g}=2.0 \mathrm{wt} \%$
Fig. 3 Stripe pattern in $\mathrm{OB}$ at $C_{c}=0.3 \mathrm{~mol} / \mathrm{L}$.

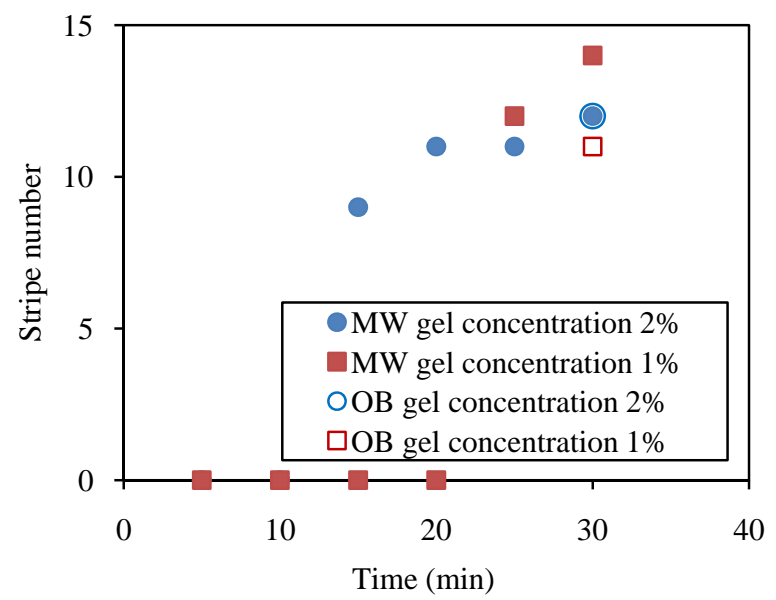

Fig. 4 Stripe number for the time at $C_{c}=0.9 \mathrm{~mol} / \mathrm{L}$.

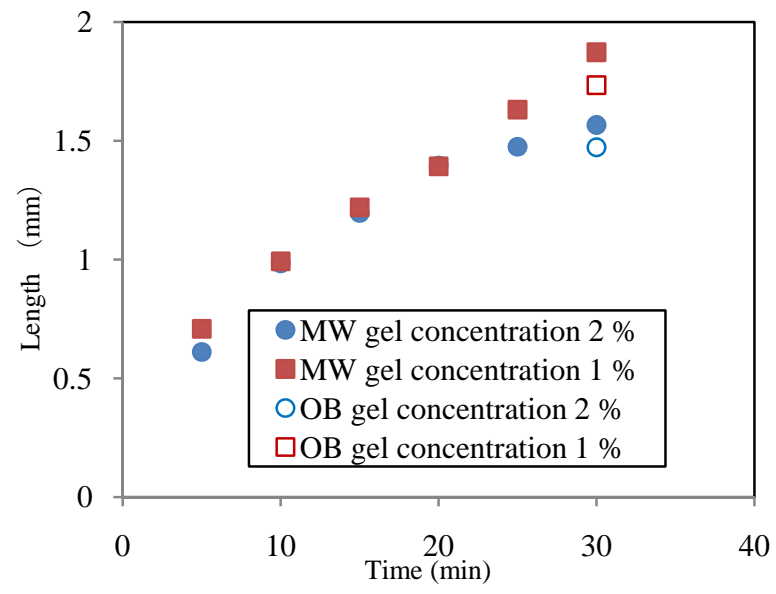

Fig. 5 Pattern length for the time at $C_{c}=0.9 \mathrm{~mol} / \mathrm{L}$. 


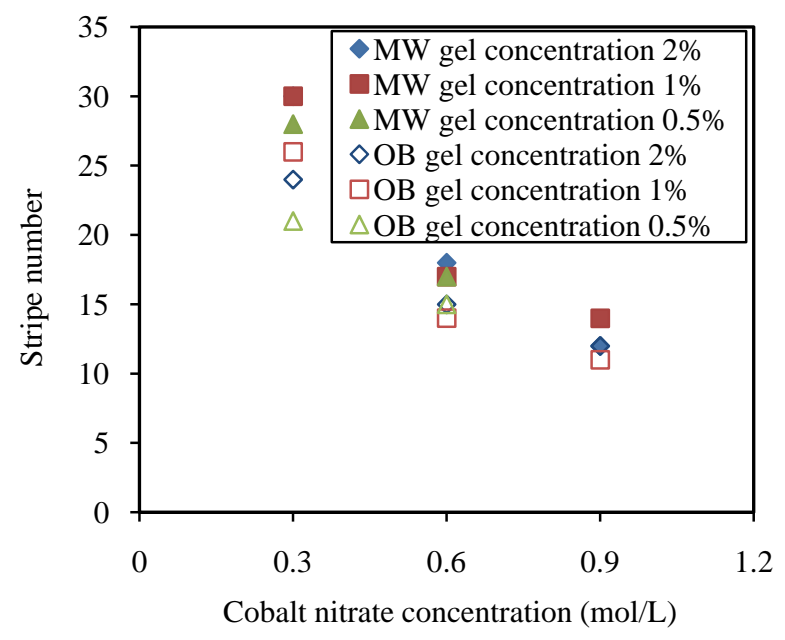

Fig. 6 Stripe number for the cobalt concentration after $30 \mathrm{~min}$.

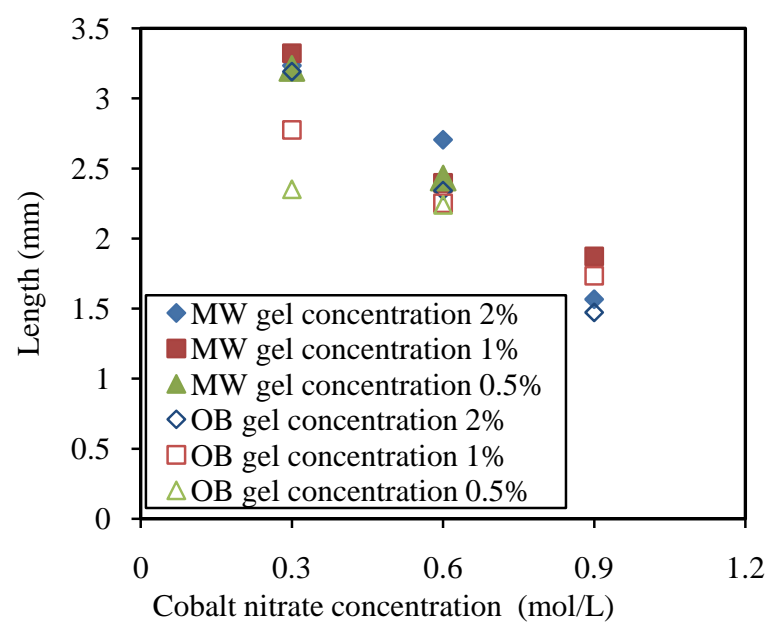

Fig. 7 Pattern length for cobalt concentration after $30 \mathrm{~min}$.

\section{Conclusions}

To understand the mechanisms of precipitation and diffusion in Liesegang patterns, experiments varying the salt and gel concentrations were conducted in an oil bath and MW reactor equipped with in situ observation system. Stripe length and number increased more quickly with respect to time under
MW irradiation than oil bath heating, and also decreased with respect to salt concentration. In particular, stronger MW effect was confirmed at lower salt condition. We explained these findings in the context of nonthermal effects (i.e., higher molecular diffusion and facilitated supersaturation) that are accelerated by radiation-induced molecular vibration. MW irradiation can modulate the diffusion and manifestation of Liesegang patterns, and may be a useful tool in other fine-scale, nonequilibrium self-organization processes that involve precipitation and nonlinearity.

\section{References}

[1] Lagzi, I.; Ueyama, D. Pattern Transition between Periodic Liesegang Pattern and Crystal Growth Regime in Reaction-Diffusion Systems. Chemical Physics Letters 2009, 468, 188-192.

[2] Toramaru, A.; Harada, T.; Okamura, T. Experimental Pattern Transitions in a Liesegang System. Physica D 2003, 183, 133-140.

[3] Sultan, R.; Ortoleva, P.; DePasquale, F.; Tartaglia, P. Bifurcation of the Ostwald-Liesegang Supersaturation-Nucleation-Depletion Cycle. EarthScience Reviews 1990, 29, 163-173.

[4] Menke, Y.; Chiaretta, D.; Milanese, D.; Ferraris, M. MW Assisted Diffusion of Au-Nanoclusters in Vitreous Silica. Journal of Non-crystalline Solids 2006, 352, 2526-2531.

[5] Clemente, R. R.; Morales, J. G. MW Precipitation of $\mathrm{CaCO}_{3}$ from Homogeneous Solutions. Journal of Crystal Growth 1996, 169, 339-346.

[6] Das, I.; Singh, P.; Agrawal, N. R.; Rastogi, R. P. Liesegang Ring Type Structures and Bifurcation in Solid-Vapor and Liquid Phase Reactions between Cobalt Nitrate and Ammonium Hydroxide. Journal of Colloid and Interface Science 1997, 192, 420-431.

[7] Msharrafieh, M.; Sultan, R. Dynamics of a Complex Diffusion-Precipitation-Re-Dissolution Liesegang Pattern. Chemical Physics Letters 2006, 421, 221-226. 